University of Minnesota Morris Digital Well

University of Minnesota Morris Digital Well

Mathematics Publications

Faculty and Staff Scholarship

1998

\title{
Timing Analysis of Targeted Hunter Searches
}

John W. Jones

Arizona State University

David P. Roberts

University of Minnesota - Morris, roberts@morris.umn.edu

Follow this and additional works at: https://digitalcommons.morris.umn.edu/mathematics

Part of the Mathematics Commons

\section{Recommended Citation}

John W. Jones and David P. Roberts. Timing Analysis of Targeted Hunter Searches. In ANTS III:

Algorithmic Number Theory. Berlin: Springer (1998), 412-423.

This Conference Proceeding is brought to you for free and open access by the Faculty and Staff Scholarship at University of Minnesota Morris Digital Well. It has been accepted for inclusion in Mathematics Publications by an authorized administrator of University of Minnesota Morris Digital Well. For more information, please contact skulann@morris.umn.edu. 


\title{
Timing Analysis of Targeted Hunter Searches
}

\author{
John W. Jones ${ }^{1}$ and David P. Roberts ${ }^{2}$ \\ 1 Department of Mathematics, Arizona State University, Box 871804 \\ Tempe, AZ 85287 \\ jj@asu.edu \\ 2 Department of Mathematics, Hill Center, Rutgers University \\ New Brunswick, NJ 08903 \\ davrobts@math.rutgers.edu
}

\begin{abstract}
One can determine all primitive number fields of a given degree and discriminant with a finite search of potential defining polynomials. We develop an asymptotic formula for the number of polynomials which need to be inspected which reflects both archimedean and non-archimedean restrictions placed on the coefficients of a defining polynomial.
\end{abstract}

Several authors have used Hunter's theorem to find a defining polynomial

$$
x^{n}+a_{1} x^{n-1}+\cdots+a_{n-1} x+a_{n} \in \mathbf{Z}[x]
$$

for each primitive degree $n$ field of absolute discriminant $D$ less than or equal to some cutoff $\Delta$. The method requires a computer search over all vectors $\left(a_{1}, \ldots, a_{n}\right)$ satisfying certain bounds.

In [JR1] we explained that one is sometimes particularly interested in the fields with $D=\Delta$, especially when all primes dividing $D$ are very small. To find just these fields by a Hunter search, one imposes not only archimedean inequalities on the $a_{i}$ as above, but also $p$-adic inequalities for each prime $p$ dividing $D$. This is an example of a targeted search, the target being $D$.

In this paper we investigate the search volume of such Hunter searches, which approximates the number of polynomials one is required to inspect. We find that these search volumes have the form

$$
\begin{aligned}
& \text { Search Volume }_{n}(D \leq \Delta)= \\
& \text { Search Volume }_{n}(D=\Delta)=\left(\prod_{p^{d} \| D} C\left(n, p^{d}\right)\right) \\
& C(n, \infty) \Delta^{(n-2) / 4} .
\end{aligned}
$$

In Section 1 we work over $\mathbf{R}$. The constant $C(n, \infty)$ is a sum of constants $C\left(n, \infty^{d}\right)$, one for each possible signature $r+2 d=n$. We identify the constant $C\left(n, \infty^{0}\right)$ using a Selberg integral; the remaining integrals are harder and we evaluate them in the cases $n \leq 7$.

In Sections 2 and 3 we work over $\mathbf{Q}_{p}$. The constant $C\left(n, p^{d}\right)$ is a sum of constants $C\left(n, p^{d}, K\right)$, one for each possible $p$-adic completion $K$ with discriminant 
$p^{d}$. Evaluating $C\left(n, p^{d}, K\right)$ requires evaluating an Igusa integral. We evaluate a few cases exactly and get a reasonable simple upper bound in all cases.

In Sections 4 and 5 we work over Q. Section 4 describes Hunter's theorem and gives an asymptotic formula for the number of defining polynomials of a degree $n$ algebra within a given search radius. In Section 5 we prove the above search volume formulas, and discuss how our results apply in practice.

We have carried out all targeted searches for $n \leq 5$, and $D$ of the form $p^{a} q^{b}$ with $p$ and $q$ primes $\leq 19$. Complete tables are available at [J1]. Our computations here show that the enormously harder case $n=6$ is feasible too. Search results will appear at [J1] as they become available.

We now fix some notation. Let $F$ be a field of characteristic zero; typically $F=\mathbf{Q}$ or one of its completions $\mathbf{Q}_{v}$ in this paper. We work with finite dimensional $F$-algebras $K$. Here, all algebras are assumed to be separable. So, $K$ factors canonically as a product of fields, $K=\prod K_{i}$.

We will work with monic degree $n$ polynomials

$$
f(x)=x^{n}+a_{1} x^{n-1}+\cdots+a_{n-1} x+a_{n} \in F[x] .
$$

Often we think of such polynomials as simply elements $\left(a_{1}, \ldots, a_{n}\right)$ of $F^{n}$. If $f(x)$ is separable, then we call $f(x)$ a defining polynomial for the $F$-algebra $K=F[x] / f(x)$. The factorization $K=\prod K_{i}$ is induced by the factorization $f(x)=\prod f_{i}(x)$ into irreducibles, via $K_{i}=F[x] / f_{i}(x)$.

Conversely, let $K$ be an algebra and $y \in K$. Let $f_{y}(x)$ be the characteristic polynomial of $y$ acting on $K$ by multiplication. Basic algebraic facts about the map $c: K \rightarrow F^{n}$ defined by $y \mapsto f_{y}$ underlie many of our considerations. For example, $c$ induces a surjection

$$
\text { (Regular elements of } K) \rightarrow \text { (Defining polynomials for } K \text { ) }
$$

with $\operatorname{Aut}(K)$ acting freely and transitively on the fibers. This accounts for the presence of $|\operatorname{Aut}(K)|$ in many formulas.

If $f(x)=\prod_{i=1}^{n}\left(x-y_{i}\right)$ we put $D(f)=\prod_{i<j}\left(y_{i}-y_{j}\right)^{2}$ and think of $D$ as a polynomial function of the $a_{j}$, as usual. Finally, if $F \subseteq \mathbf{C}$ we let $T_{2}(f)=$ $\sum_{i=1}^{n}\left|y_{i}\right|^{2}$.

\section{Archimedean Volumes}

Let $A$ be a degree $n$ algebra over $\mathbf{R}$. So, we can simply take $A=\mathbf{R}^{r} \times \mathbf{C}^{d}$ for some $r+2 d=n$. The characteristic polynomial of $y \in A$ is

$$
f_{y}(x)=x^{n}+a_{1} x^{n-1}+\cdots+a_{n-1} x+a_{n} \in \mathbf{R}[x] .
$$

Let $A^{0}$ be the set of elements of $A$ with trace 0 , and consider the corresponding space of polynomials

$$
P^{0}(A, r)=\left\{f_{y} \in \mathbf{R}[x]: y \in A^{0} \text { and } T_{2}\left(f_{y}\right) \leq r^{2}\right\} .
$$

We measure the volume of $P^{0}(A, r)$ with respect to the usual volume form $d a_{2} \cdots d a_{n}$. 


\section{Proposition 1.1.}

$$
\operatorname{vol}\left(P^{0}(A, r)\right)=\operatorname{vol}\left(P^{0}(A, 1)\right) r^{(n+2)(n-1) / 2}
$$

Proof. One has a linear map

$$
\begin{aligned}
L: P^{0}(A, 1) & \rightarrow P^{0}(A, r) \\
\left(a_{2}, \ldots, a_{n}\right) & \mapsto\left(r^{2} a_{2}, \ldots, r^{n} a_{n}\right) .
\end{aligned}
$$

The Jacobian of this map is $r$ to the power

$$
\sum_{j=2}^{n} j=\frac{(n+2)(n-1)}{2} .
$$

This simple observation is the most important point in analyzing Hunter searches.

We work more generally with

$$
\zeta_{A}(s):=\int_{P^{0}(A, 1)}|D|^{s-\frac{1}{2}} d a_{2} \cdots d a_{n} .
$$

The desired volume $\operatorname{vol}\left(P^{0}(A, 1)\right)$ is just the special value $\zeta_{A}(1 / 2)$. A general formula for $\zeta_{A}(s)$ would be desirable, since it would give one the moments of the polynomial discriminants encountered in a Hunter search. For example, to compute the average polynomial discriminant encountered one needs the number $\zeta_{A}(3 / 2)$, as well as $\zeta_{A}(1 / 2)$.

Let $A^{0}(1)$ be the unit ball in $A^{0}$; it is a degree $|\operatorname{Aut}(A)|$ cover of $P^{0}(A, 1)$ via the characteristic polynomial map $c$. One can pull back the defining integral to $A^{0}(1)$; at this step the Jacobian $|D|^{1 / 2}$ enters the integrand. One can next extend the integral to the full unit ball $A(1)$. Using the homogeneity of the integrand, one can replace the sharp radial cutoff $\rho \leq 1$ by an integral over all of $A$ against a Gaussian $e^{-\rho^{2} / 2}$. The net result is

$$
\zeta_{A}(s)=\frac{2^{n(s-n s-1) / 2}}{|\operatorname{Aut}(A)| \sqrt{\pi n}\left(\frac{(n s+1)(n-1)}{2}\right) !} \int_{A} e^{-\rho^{2} / 2}|D|^{s} \omega .
$$

Here $\omega$ is the standard volume form on $A$, giving the unit ball $\rho \leq 1$ its usual volume $\pi^{n / 2} /(n / 2)$ !. Also $\left|\operatorname{Aut}\left(\mathbf{R}^{r} \times \mathbf{C}^{d}\right)\right|=r ! d ! 2^{d}$.

\section{Proposition 1.2.}

$$
\operatorname{vol}\left(P^{0}\left(\mathbf{R}^{n}, 1\right)\right)=\frac{2^{-n(n-5) / 4} \prod_{j=1}^{n}(j / 2) !}{n ! \sqrt{\pi n}\left(\frac{(n+2)(n-1)}{4}\right) !}
$$


Proof. In the case $A=\mathbf{R}^{n}$ the roots $y_{1}, \ldots, y_{n}$ are coordinates on $A$ and $\omega=$ $d y_{1} \cdots d y_{n}$. A special case of Selberg's integral is

$$
\int_{-\infty}^{\infty} \cdots \int_{-\infty}^{\infty} e^{-r^{2} / 2}|D|^{s} d y_{1} \cdots d y_{n}=(2 \pi)^{n / 2} \prod_{j=1}^{n} \frac{(j s) !}{s !} ;
$$

see e.g. [M1], 17.6.7. Evaluating at $s=1 / 2$ this becomes $2^{3 n / 2} \prod_{j=1}^{n}(j / 2)$ !, yielding the proposition.

Proposition 1.3. The ratios $\operatorname{vol}\left(P^{0}\left(\mathbf{R}^{r} \times \mathbf{C}^{d}, 1\right)\right) / \operatorname{vol}\left(P^{0}\left(\mathbf{R}^{n}, 1\right)\right)$ for $n \leq 7$ are as follows.

\begin{tabular}{r|rrrrr}
$d \backslash n$ & 3 & 4 & 5 & 6 & 7 \\
\hline 0 & 1 & 1 & 1 & 1 & 1 \\
1 & 5 & 18 & 58 & 179 & 543 \\
2 & & 9 & $134 \frac{1}{3}$ & 1355 & 11875 \\
3 & & & & $451 \frac{2}{3}$ & $17466 \frac{1}{3}$
\end{tabular}

Proof. Let

$$
I=\prod_{1 \leq i<j \leq n}\left(y_{i}-y_{j}\right)
$$

be the indicated square root of $D$. We need to compute

$$
\int_{\mathbf{R}^{r} \times \mathbf{C}^{d}} e^{-\rho^{2} / 2}|I| \omega
$$

Taking $y_{r+1}, \ldots, y_{r+d}$ as coordinates on $\mathbf{C}^{d}$ and writing $y_{k}=\left(u_{k}+i v_{k}\right) / \sqrt{2}$ one has

$$
\begin{aligned}
\rho^{2} & =\sum_{j=1}^{r} y_{j}^{2}+\sum_{k=1}^{d}\left(u_{k}^{2}+v_{k}^{2}\right) \\
\omega & =d y_{1} \cdots d y_{r} d u_{1} \cdots d u_{d} d v_{1} \cdots d v_{d} \\
|I| & =f\left(y_{1}, \ldots, y_{r}, u_{1}, \ldots, u_{d}, v_{1}, \ldots, v_{d}\right) \prod_{1 \leq i<j \leq r}\left|y_{i}-y_{j}\right| \prod_{k=1}^{d}\left|v_{k}\right|
\end{aligned}
$$

with $f$ a polynomial. One can expand $f$ and integrate out the $u_{k}$ 's and the $v_{k}$ 's using

$$
\int_{0}^{\infty} e^{-x^{2} / 2} x^{j} d x=2^{(j-1) / 2}\left(\frac{j-1}{2}\right) !
$$

$2 d$ times on each term. One is left with an integral of the form

$$
\int_{-\infty}^{\infty} \cdots \int_{-\infty}^{\infty} e^{-\left(y_{1}^{2}+\ldots+y_{r}^{2}\right) / 2}\left(\prod_{1 \leq i<j \leq r}\left|y_{i}-y_{j}\right|\right) g\left(y_{1}, \ldots, y_{r}\right) d y_{1} \cdots d y_{r}
$$

with $g\left(y_{1}, \ldots, y_{r}\right)$ a symmetric polynomial in the $y_{i}$. Here the absolute values pose a problem. For $r \leq 3$ this obstruction can be surmounted in an elementary way and one can again integrate term-by-term. When $d=1$ the moment formulas in [M1], Section 17.8 suffice. This covers all cases with $n \leq 7$. 
The case of cubics $f(x)=x^{3}+a_{2} x+a_{3}$ is illustrative. The two regions $P^{0}(A, 1)$ are shown in Figure 1. In the case $A=\mathbf{R} \times \mathbf{C}$, let $r^{2}=y_{1}^{2}+\left|y_{2}\right|^{2}+\left|y_{3}\right|^{2}$

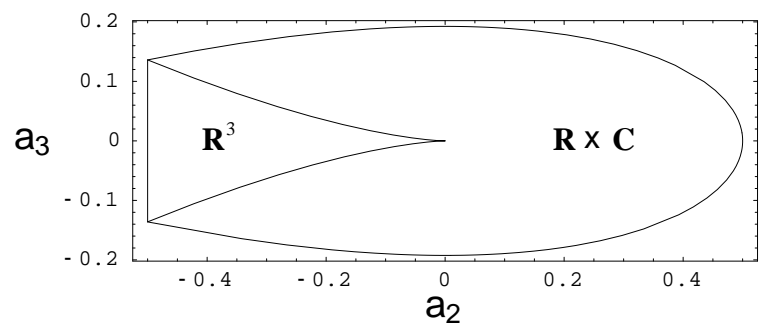

Fig. 1. The sets $P^{0}(A, 1)$ for cubics $f(x)=x^{3}+a_{2} x+a_{3}$

and express the real root $y_{1}$ as $r t$. Then the defining integral for $\zeta_{\mathbf{R} \times \mathbf{C}}(s)$ can be evaluated by changing variables from $\left(a_{2}, a_{3}\right)$ to $0 \leq r \leq 1,-\sqrt{2 / 3} \leq t \leq \sqrt{2 / 3}$. The result is

$$
\zeta_{\mathbf{R} \times \mathbf{C}}(s)=\frac{\sqrt{\pi} 2^{-s-1} 3^{2 s-\frac{1}{2}}(s-1 / 2) !}{(1+3 s) s !}{ }_{2} F_{1}\left(-2 s, \frac{1}{2}+s ; 1+s ; \frac{2}{3}\right) .
$$

The presence of the hypergeometric function ${ }_{2} F_{1}$ indicates that the general $\zeta_{\mathbf{R}^{r} \times \mathbf{C}^{d}}(s)$ is more complicated than the general $\zeta_{\mathbf{R}^{n}}(s)$.

\section{Ultrametric Masses}

The set of isomorphism classes of degree $n$ algebras $A$ over $\mathbf{Q}_{p}$ is much more complicated than in the archimedean case $v=\infty$. A starting point for analyzing this set is a mass formula, due to Krasner and Serre [S1]. Here is a quick summary, more details being contained in [R2].

The mass of $A$ is by definition $m_{A}:=1 /|\operatorname{Aut}(A)|$. Let $\mathbf{Q}_{p}^{\text {un }}$ be a maximal unramified extension of $\mathbf{Q}_{p}$ and put $A^{\text {un }}=A \otimes \mathbf{Q}_{p}^{\text {un }}$. Call two algebras $A_{1}$ and $A_{2}$ geometrically equivalent if $A_{1}^{\text {un }} \cong A_{2}^{\text {un }}$. Then, the sum of $m_{A}$ over $A$ in a geometric equivalence class is 1 .

Let $m_{n, p^{d}}$ be the sum of $m_{A}$ over all totally ramified $A$ of degree $n$ and discriminant $p^{d}$. Then, in the tame case $p \nmid n$, the only non-vanishing $m_{n, p^{d}}$ is $m_{n, p^{n-1}}=1$. The first few wild cases are shown in Table 1. Other wild cases are more complicated, but are also governed by the Krasner-Serre mass formula $\sum_{d} m_{n, p^{d}} p^{n-1-d}=1$.

The general case reduces to the totally ramified case just summarized. Given $A$, from the canonical factorization $A^{\text {un }}=\prod A_{i}^{\text {un }}$ one gets an unordered collection of $\left(n_{i}, d_{i}\right)$. The sum of $m_{A}$ over algebras $A$ giving rise to these $\left(n_{i}, d_{i}\right)$ is $\prod m_{n_{i}, p_{i}^{d_{i}}}$. In particular, the degree partition $\lambda_{A}=\left(n_{1}, n_{2}, \ldots\right)$ is a complete geometric invariant of a tame algebra. 
Table 1. Masses for low degree wildly ramified algebras

$m_{p, p^{d}}=\left\{\begin{array}{lr|rrr}p-1 \text { if } p \leq d \leq 2 p-2 & d & m_{4,2^{d}} & m_{6,2^{d}} & m_{6,3^{d}} \\\right.$\cline { 2 - 5 } & 4 & 1 & & \\ $p \quad \text { if } d=2 p-1 & 5 & & & \\ 0 \quad \text { else } & 7 & & 1 & 2 \\ & 8 & 4 & 2 & \\ & 9 & 4 & & 6 \\ & 10 & 4 & 4 & 6 \\ & 11 & 8 & 8 & 9\end{array}$

Let $M\left(n, p^{d}\right)$ be the sum of $m_{A}$ over all $p$-adic algebras with degree $n$ and discriminant $p^{d}$. The above discussion is sufficient for computing $M\left(n, p^{d}\right)$ for $n \leq 7$. The integers $M\left(n, p^{d}\right)$ appear in Corollary 3.3 and also the table in Section 5 .

\section{Ultrametric Volumes}

Let $A$ be a degree $n$ algebra over $\mathbf{Q}_{p}$, with ring of integers $\mathcal{O}$, and discriminant $p^{d_{A}}$. Define

$$
P(A)=\left\{f_{y}(x) \in \mathbf{Z}_{p}[x]: y \in \mathcal{O}\right\} .
$$

We measure volumes with $d a_{1} \cdots d a_{n}$, so that all of $\mathbf{Z}_{p}^{n}$ gets volume 1 .

Proposition 3.1. With $Z_{A}(t)$ as defined in the proof below,

(i) $\operatorname{vol}(P(A))=\frac{Z_{A}(1 / p)}{|\operatorname{Aut}(A)| p^{d_{A}}}$

(ii) $Z_{A}(1 / p) \leq 1$.

Proof. Let $\omega$ be Haar measure on $A$, normalized so that $\omega(\mathcal{O})=1$. We use the characteristic polynomial map $c: \mathcal{O} \rightarrow P(A)$. Pulled back to $\mathcal{O}$, the polynomial discriminant function $D$ factors as $p^{d_{A}} I^{2}$. Here, $I$ is a polynomial function on $\mathcal{O}$ with $\mathbf{Z}_{p}$ coefficients. Note, $\mathbf{Z}_{p}[y]$ has index $1 /|I(y)|_{p}$ in $\mathcal{O}$.

The Jacobian function $c^{*}\left(d a_{1} \cdots d a_{n}\right) / \omega$ is $p^{-d_{A}}|I|_{p}$. On regular elements, i.e. elements on which $I$ is non-zero, $c$ has degree $\operatorname{Aut}(A)$. So

$$
\begin{aligned}
\zeta_{A}(s):=\int_{P(A)}|D|_{p}^{s-\frac{1}{2}} d a_{1} \cdots d a_{n} & =\frac{1}{|\operatorname{Aut}(A)|} \int_{\mathcal{O}}\left|p^{d_{A}} I^{2}\right|_{p}^{s-\frac{1}{2}} p^{-d_{A}}|I|_{p} \omega \\
& =\frac{1}{|\operatorname{Aut}(A)| p^{d_{A}\left(\frac{3}{2}-s\right)}} \int_{\mathcal{O}}|I|_{p}^{2 s} \omega \\
& =\frac{Z_{A}\left(p^{-2 s}\right)}{|\operatorname{Aut}(A)| p^{d_{A}\left(\frac{3}{2}-s\right)}} .
\end{aligned}
$$


Here we have defined

$$
Z_{A}(t)=\sum_{j=0}^{\infty} \omega(\mathcal{O}[j]) t^{j}
$$

with $\mathcal{O}[j]$ the set of $y \in \mathcal{O}$ with $|I(y)|_{p}=1 / p^{j}$. Plugging in $s=1 / 2$ gives part (i).

To prove part $(i i)$, we note that $Z_{A}(t)$ is a power series with positive coefficients such that $Z_{A}(1)=\omega(\mathcal{O})=1$. It is an increasing function on $[0,1]$ and so $Z_{A}(1 / p) \leq 1$.

The function $Z_{A}(t)$ is an example of an Igusa zeta function [D1]. Thus, it is known to be in $\mathbf{Q}(t)$.

Proposition 3.1 and its proof make no reference to the classification of $p$-adic algebras sketched in Section 2. Define

$$
\begin{aligned}
P(\lambda) & =\bigcup_{\lambda_{A}=\lambda} P(A) \\
P\left(n, p^{d}\right) & =\bigcup_{d_{A}=d} P(A) .
\end{aligned}
$$

Summing over $A$ with $\lambda_{A}=\lambda$ in Proposition 3.1 and using the Krasner-Serre mass formula gives Corollary 3.2 below. Summing over $A$ with $d_{A}=d$ in Proposition 3.1 and using the definition of $M\left(n, p^{d}\right)$ gives Corollary 3.3.

Corollary 3.2. For $\lambda$ a partition of $n$,

$$
\operatorname{vol}(P(\lambda)) \leq \frac{1}{p^{n-\ell(\lambda)}} .
$$

where $\ell(\lambda)$ denotes the length of $\lambda$.

Corollary 3.3. For $d \in \mathbf{Z}_{\geq 0}$,

$$
\operatorname{vol}\left(P\left(n, p^{d}\right)\right) \leq \frac{M\left(n, p^{d}\right)}{p^{d}} .
$$

On the other hand, one can also prove Corollary 3.2 directly, using neither the Krasner-Serre mass formula, nor Proposition 3.1.

Direct proof of Corollary 3.2. Write $\lambda=\left(\lambda_{1}, \ldots, \lambda_{\ell(\lambda)}\right)$, with each $\lambda_{i}>0$. Let $P(\lambda)_{1} \subset \mathbf{F}_{p}^{n}$ be the reduction of $P(\lambda) \subset \mathbf{Z}_{p}^{n}$. For $e$ a positive integer, let $\mu_{e}$ be the number of $i$ such that $\lambda_{i}=e$. Very simply,

$$
P(\lambda)_{1}=\left\{\prod_{e} f_{e}(x)^{e}\right\}
$$

where $f_{e}(x) \in \mathbf{F}_{p}[x]$ is monic of degree $\mu_{e}$. To give an element of $P(\lambda)_{1}$ is to give the coefficients of the $f_{e}$. There are $\sum \mu_{e}=\ell(\lambda)$ coefficients, and so $P(\lambda)_{1}$ is $p^{\ell(\lambda)} / p^{n}$ of $\mathbf{F}_{p}^{n}$. 
It would be nice to compute $Z_{A}(1 / p)$ exactly. To do this it seems necessary to compute all of $Z_{A}(t)$. We have succeeded when $n$ is prime and $A$ is a field; the results in the unramified case $U$ and the totally ramified case $R$ are

$$
Z_{U}(t)=\frac{1-\frac{1}{p^{n-1}}}{1-\frac{t^{n(n-1) / 2}}{p^{n-1}}} \quad Z_{R}(t)=\frac{\left(1-\frac{1}{p}\right)\left(1-\frac{t^{(n-1)^{2} / 2}}{p^{n-1}}\right)}{\left(1-\frac{t^{(n-1) / 2}}{p}\right)\left(1-\frac{t^{n(n-1) / 2}}{p^{n-1}}\right)}
$$

We have also computed several more difficult $Z_{A}(t)$, sometimes directly, and sometimes making use of the stationary phase formula [D1], Theorem 3.4. The resulting formulas are quite complicated.

\section{The Search Set}

Let $K$ be a degree $n$ algebra over $\mathbf{Q}$ with absolute discriminant $D$. With respect to the quadratic form $T_{2}$, one has an orthogonal decomposition $K=K^{0} \oplus \mathbf{Q}$, $K^{0}$ being the subspace of traceless elements.

Let $\mathcal{O}$ be the ring of integers in $K$. Let $\mathcal{O}^{\prime}$ be the projection of $\mathcal{O}$ to $K^{0}$. As a lattice in the Euclidean space $K^{0} \otimes \mathbf{R}, \mathcal{O}^{\prime}$ has covolume $\sqrt{D / n}$.

Let $g_{m}$ be the smallest real number so that every lattice in Euclidean space $\mathbf{R}^{m}$ with covolume $V$ has a non-zero vector of length $\leq\left(g_{m} V^{2}\right)^{1 /(2 m)}$. The value of $g_{m}$ is known ([CS1], Table 1.2) for $m \leq 8$.

\begin{tabular}{c|cccccccc}
$m$ & 1 & 2 & 3 & 4 & 5 & 6 & 7 & 8 \\
\hline$g_{m}$ & 1 & $1 \frac{1}{3}$ & 2 & 4 & 8 & $21 \frac{1}{3}$ & 64 & 256
\end{tabular}

In the literature one often sees Hermite's constant $\gamma_{m}=\sqrt[m]{g_{m}}$ instead of $g_{m}$.

Define

$$
r_{D}=\left(\frac{g_{n-1} D}{n}\right)^{1 /(2 n-2)} .
$$

One gets immediately that in $\mathcal{O}^{\prime}$ there is a non-zero vector $y^{\prime}$ of length $\leq r_{D}$. The subalgebra $\mathbf{Q}\left(y^{\prime}\right)$ of $K$ strictly contains $\mathbf{Q}$; so if $K$ is a primitive field, $\mathbf{Q}\left(y^{\prime}\right)$ is automatically all of $K$.

Henceforth in this paper we take $n \geq 3$ to avoid trivialities. By replacing $y^{\prime}$ by $-y^{\prime}$ one can assume that $a_{3}^{\prime} \geq 0$ in its characteristic polynomial. As $j$ varies from 0 to $n-1$, exactly one of $y=y^{\prime}-j / n$ is in $\mathcal{O}$. This element $y$ has characteristic polynomial $f_{y} \in P\left(r_{D}\right)$; here the search set $P(r)$ is the set of polynomials

$$
f(x)=\prod_{i=1}^{n}\left(x-y_{i}\right)=x^{n}+a_{1} x^{n-1}+\cdots+a_{n-1} x+a_{n} \in \mathbf{Z}[x]
$$

satisfying the two conditions 
(i) trace condition:

$$
a_{1} \in\{0, \ldots, n-1\} \text { and } a_{3} \geq \frac{(n-2) a_{1} a_{2}}{n}-\frac{(n-1)(n-2) a_{1}^{3}}{3 n^{2}}
$$

(ii) length condition:

$$
T_{2}(f) \leq \frac{a_{1}^{2}}{n}+r^{2}
$$

Proposition 4.1. Let $K$ be a degree $n$ algebra over $\mathbf{Q}$ with absolute discriminant $D$. Let $m(K, \Delta)$ be the number of defining polynomials for $K$ in $P\left(r_{\Delta}\right)$.

(i) If $K$ is a primitive field and $D \leq \Delta$, then $m(K, \Delta) \geq 1$.

(ii) For general $K$,

$$
m(K, \Delta) \sim \frac{m_{n}}{|\operatorname{Aut}(K)|} \sqrt{\frac{\Delta}{D}} \quad \text { with } \quad m_{n}=\frac{\sqrt{g_{n-1}} \pi^{(n-1) / 2}}{2\left(\frac{n-1}{2}\right) !}
$$

as $\Delta \rightarrow \infty$.

Proof. Part (i) is essentially Hunter's theorem, see e.g. [C1], Theorem 6.4.1. It is proved by our discussion above. Our trace condition is a modification of the standard one. We make this modification in order to fully exploit the involution $y^{\prime} \mapsto-y^{\prime}$, thereby making $|P(r)|$ as small as possible.

For Part (ii), let $\mathcal{O}_{\Delta,+}^{\prime}$ be the subset of $\mathcal{O}^{\prime}$ consisting of elements $y^{\prime}$ with length $\leq r_{\Delta}$ and $a_{3}^{\prime} \geq 0$. Let $\mathcal{O}_{\text {reg, }, \Delta,+}^{\prime}$ be the subset of $\mathcal{O}_{\Delta,+}^{\prime}$ consisting of regular elements. Then

$$
\begin{aligned}
|\operatorname{Aut}(K)| m(K, \Delta) & =\left|\mathcal{O}_{\text {reg, }, \Delta,+}^{\prime}\right| \sim\left|\mathcal{O}_{\Delta,+}^{\prime}\right| \sim \frac{\left(\text { Volume of ball of radius } r_{\Delta}\right)}{2\left(\text { Covolume of } \mathcal{O}^{\prime}\right)} \\
& =\frac{\left(r_{\Delta} \sqrt{\pi}\right)^{n-1} /\left(\frac{n-1}{2}\right) !}{2 \sqrt{D / n}}=\frac{\sqrt{g_{n-1}} \pi^{(n-1) / 2}}{2\left(\frac{n-1}{2}\right) !} \sqrt{\frac{\Delta}{D}}
\end{aligned}
$$

as $\Delta \rightarrow \infty$.

Part (ii) relates to the phenomenon that searches tend to find several defining polynomials for each primitive field sought, as well as defining polynomials for non-primitive fields. For $3 \leq n \leq 9$ one has

\begin{tabular}{c|ccccccc}
$n$ & 3 & 4 & 5 & 6 & 7 & 8 & 9 \\
\hline$m_{n}$ & 1.8 & 3.0 & 4.9 & 7.4 & 11.9 & 18.9 & 32.5
\end{tabular}

to one decimal place. 


\section{Timing Analysis}

To incorporate targeting into the formalism, let $S$ be a finite set of places of $\mathbf{Q}$ containing $\infty$. For $v \in S$, let $A_{v}$ be a degree $n$ algebra over $\mathbf{Q}_{v}$. Let

$$
P\left(\left\{A_{v}\right\}, r\right)=\left\{f(x) \in P(r): \mathbf{Q}_{v}[x] / f(x) \cong A_{v} \text { for } v \in S\right\} .
$$

From Proposition 4.1, $P\left(\left\{A_{v}\right\}, r_{\Delta}\right)$ contains a defining polynomial for every primitive degree $n$ field $K$ with absolute discriminant $D \leq \Delta$ and $K_{v} \cong A_{v}$, $v \in S$.

\section{Proposition 5.1.}

$$
\left|P\left(\left\{A_{v}\right\}, r_{\Delta}\right)\right| \sim \frac{n}{2}\left(\frac{g_{n-1}}{n}\right)^{(n+2) / 4} \operatorname{vol}\left(P^{0}\left(A_{\infty}, 1\right)\right)\left(\prod_{p} \operatorname{vol}\left(P\left(A_{p}\right)\right)\right) \Delta^{(n+2) / 4}
$$

as $\Delta \rightarrow \infty$.

Proof. For elements in $P\left(\left\{A_{\infty}\right\}, r_{\Delta}\right)$ there are $n$ possible values of $a_{1}$, each giving asymptotically the same number of polynomials; this accounts for the factor $n$. Those with $a_{1}=0$ are the intersection of the standard lattice $\mathbf{Z}^{n-1}$ with the interior of the region $P^{0}\left(A_{\infty}, r_{\Delta}\right)_{+}$in $\mathbf{R}^{n-1}$. The + indicates the extra condition $a_{3} \geq 0$, and accounts for the 2 in the denominator. Proposition 1.1 and the definition of $r_{\Delta}$ account for the factor $\operatorname{vol}\left(P^{0}\left(A_{\infty}, r_{\Delta}\right)\right)=$ $\left(g_{n-1} \Delta / n\right)^{(n+2) / 4} \operatorname{vol}\left(P^{0}\left(A_{\infty}, 1\right)\right)$. Finally the ultrametric conditions account for the extra factors $\operatorname{vol}\left(P\left(A_{p}\right)\right)$.

The deeper Propositions 1.2 and 1.3 determine the archimedean volumes for $n \leq 7$; Proposition 3.1 bounds the ultrametric volumes in general.

Proposition 5.1 is an asymptotic formula. However one would expect, and experience shows, that it applies well when $\Delta$ is simply the product of the discriminants of the $A_{p}$ 's. In this restricted context, and summing over $p$-adic algebras with a given discriminant, the formula can be restated as follows. Define

$$
\begin{aligned}
C\left(n, \infty^{d}\right) & =\frac{n}{2}\left(\frac{g_{n-1}}{n}\right)^{(n+2) / 4} \operatorname{vol}\left(P^{0}\left(\mathbf{R}^{n-2 d} \times \mathbf{C}^{d}, 1\right)\right) \\
C\left(n, p^{d}\right) & =\operatorname{vol}\left(P\left(n, p^{d}\right)\right) p^{d} .
\end{aligned}
$$

Then, a Hunter search for all primitive fields of signature $\left(n-2 d_{\infty}, d_{\infty}\right)$ and absolute discriminant $\prod p^{d_{p}}$ requires inspection of approximately

$$
C\left(n, \infty^{d_{\infty}}\right) \prod_{p} C\left(n, p^{d_{p}}\right) p^{d_{p}(n-2) / 4}
$$

polynomials. (Naturally there are no such fields unless $(-1)^{d_{\infty}} \prod p^{d_{p}}$ is congruent to 0 or 1 modulo 4 . If $n=6$, some searches can be replaced by easier searches via sextic twinning $[\mathrm{R} 1]$.

In the literature there are several methods which allow one to implement the length inequality and target the local algebra at $\infty$ with little loss [BFP1], 
[SPD1], [O1], [DO1]. In principal, $p$-adic bounds on the $a_{i}$ giving only the slight loss $C\left(n, p^{d}\right) \leq M\left(n, p^{d}\right)$ of Corollary 3.3 are easy to describe since they amount to collections of congruences on the $a_{i}$. For example, in the tame case one can follow the direct proof of Corollary 3.2. In practice, for large $p$ and/or $n$ it can become unwieldy to implement sharp $p$-adic bounds as well.

Table 2 below gives what we call local difficulty ratings, namely the numbers $\log _{10}\left(C\left(n, \infty^{d}\right)\right)$ and $\log _{10}\left(M\left(n, p^{d}\right) p^{d(n-2) / 4}\right)$. All entries are rounded to the nearest tenth. The rows labelled All give totals for all values of $d$.

Table 2. Local Difficulty Ratings

\begin{tabular}{|r|ccccccc|ccccccc|}
\hline$d$ & $\infty$ & 2 & 3 & 5 & 7 & 11 & 13 & $\infty$ & 2 & 3 & 5 & 7 & 11 & 13 \\
\hline 0 & -3.1 & & \multicolumn{3}{c}{ Quartics } & & -5.3 & & & Quintics & & \\
1 & -1.9 & - & 0.2 & 0.3 & 0.4 & 0.5 & 0.6 & -3.5 & - & 0.4 & 0.5 & 0.6 & 0.8 & 0.8 \\
2 & -2.2 & 0.6 & 0.5 & 1.0 & 1.1 & 1.3 & 1.4 & -3.1 & 0.8 & 0.7 & 1.3 & 1.6 & 1.9 & 2.0 \\
3 & & 0.8 & 1.2 & 1.0 & 1.3 & 1.6 & 1.7 & & 1.0 & 1.6 & 1.9 & 2.2 & 2.6 & 2.8 \\
4 & & 0.9 & 1.3 & & & & & & 1.5 & 2.1 & - & 2.5 & 3.1 & 3.3 \\
5 & & 1.1 & 1.7 & & & & & & 1.7 & 2.5 & 3.2 & & & \\
6 & & 1.7 & & & & & & & 2.1 & 2.6 & 3.7 & & & \\
7 & & - & & & & & & & - & & 4.3 & & & \\
8 & & 1.8 & & & & & & & 2.4 & & 4.8 & & & \\
9 & & 2.0 & & & & & & & 2.6 & & 5.4 & & & \\
10 & & 2.1 & & & & & & & 2.9 & & & & & \\
11 & & 2.6 & & & & & & & 3.4 & & & & & \\
All & -1.7 & 2.9 & 1.9 & 1.4 & 1.5 & 1.8 & 1.9 & -3.0 & 3.6 & 3.0 & 5.5 & 2.7 & 3.3 & 3.5 \\
\hline 0 & -8.1 & & & Sextics & & & -11.4 & & & Septics & & \\
1 & -5.9 & - & 0.5 & 0.7 & 0.8 & 1.0 & 1.1 & -8.7 & - & 0.6 & 0.9 & 1.1 & 1.3 & 1.4 \\
2 & -5.0 & 0.9 & 1.0 & 1.7 & 2.0 & 2.4 & 2.5 & -7.3 & 1.1 & 1.2 & 2.0 & 2.4 & 2.9 & 3.1 \\
3 & -5.5 & 1.2 & 2.0 & 2.6 & 3.0 & 3.6 & 3.8 & -7.2 & 1.4 & 2.4 & 3.1 & 3.6 & 4.4 & 4.7 \\
4 & & 1.9 & 2.7 & 3.1 & 3.9 & 4.6 & 4.9 & & 2.2 & 3.2 & 4.0 & 4.8 & 5.8 & 6.2 \\
5 & & 2.1 & 3.1 & 4.2 & 4.2 & 5.2 & 5.6 & & 2.5 & 3.9 & 5.1 & 5.8 & 7.0 & 7.4 \\
6 & & 2.8 & 3.8 & 4.8 & & & & & 3.4 & 4.7 & 6.2 & - & 7.8 & 8.4 \\
7 & & 2.7 & 4.1 & 5.5 & & & & & 3.5 & 5.2 & 7.0 & 8.2 & & \\
8 & & 3.5 & 4.8 & 6.2 & & & & & 4.3 & 5.9 & 7.9 & 9.2 & & \\
9 & 3.9 & 5.4 & 7.0 & & & & & 4.6 & 6.4 & 8.8 & 10.3 & & \\
10 & & 4.1 & 5.9 & & & & & & 5.0 & 7.1 & 9.4 & 11.3 & & \\
11 & & 4.8 & 6.2 & & & & & & 5.6 & 7.5 & & 12.4 & & \\
12 & & 4.7 & & & & & & & 5.7 & & & 13.5 & & \\
13 & & 5.1 & & & & & & & 6.3 & & & 14.6 & & \\
14 & & 5.4 & & & & & & & 6.5 & & & & & \\
All & -4.8 & 5.7 & 6.4 & 7.1 & 4.4 & 5.3 & 5.7 & -6.9 & 6.8 & 7.7 & 9.5 & 14.6 & 7.9 & 8.4 \\
\hline
\end{tabular}

The translation from search volumes to search times requires that one incorporate a number of practical concerns as well. All told, one can expect that a degree $n$ search with difficulty $x$ will take longer than a degree $n-1$ search with 
difficulty $x$. For our current programs, in degrees 5 and 6 on a medium speed personal computer, the translation from volumes to times goes as follows.

For quintics, searches with difficulty rating $x$ take us about $10^{x-7.9}$ days. For example, the search for all $2^{11} 3^{6} 5^{9}$ quintics has difficulty rating $-3.0+3.4+$ $2.6+5.4=8.4$ and took $10^{0.5} \approx 3$ days.

For sextics, searches with difficulty rating $x$ take us about $10^{x-7.3}$ days. For example, the search for all primitive $2^{14} 5^{9}$ sextics has difficulty rating $-4.8+$ $5.4+7.0=7.6$ and took around $10^{0.3} \approx 2$ days.

In [JR1] we found all sextic fields ramified within $S=\{\infty, 2,3\}$. Table 2 shows that a few other $\{\infty, p, q\}$ are easier, while harder cases like $\{\infty, 3,5\}$ are feasible too.

\section{References}

[BFP1] Buchmann, J., Ford, D., and Pohst, M., Enumeration of quartic fields of small discriminant, Math. Comp. 61 (1993) 873-879.

[C1] Cohen, H.: A Course in Computational Algebraic Number Theory, GTM 138 Springer Verlag, 1995.

[CS1] Conway, J. and Sloane, N.: Sphere Packings, Lattices, and Groups, Springer Verlag, 1988.

[D1] Denef, J.: Report on Igusa's local zeta function, Séminaire Bourbaki 741, Astérisque 201-202-203, 359-386.

[DO1] Diaz y Diaz, F. and Olivier, M., Imprimitive ninth-degree number fields with small discriminants, Math. Comp. 64 (1995) 305-321.

[J1] Jones, J.: Tables of number fields with prescribed ramification, a WWW site, http://math.la.asu.edu/ ${ }^{j} j /$ numberfields

[JR1] Jones, J. and Roberts, D.: Sextic number fields with discriminant $-{ }^{j} 2^{a} 3^{b}$, to appear in the Proceedings of the Fifth Conference of the Canadian Number Theory Association.

[M1] Mehta, M.: Random Matrices, $2^{\text {nd }}$ edition, Academic Press, 1991.

[O1] Olivier, M., The computation of sextic fields with a cubic subfield and no quadratic subfield, Math. Comp. 58 (1992) 419-432.

[R1] Roberts, D.: Twin sextic algebras, to appear in Rocky Mountain J. Math.

[R2] Roberts, D.: Low degree p-adic fields, in preparation.

[S1] Serre, J.-P.: Une "formule de masse" pour les extensions totalement ramifiées de degré donné d'un corps local, C. R. Acad. Sci. Paris Sér. A-B 286 (1978), no. 22, A1031-A1036.

[SPD1] Schwarz, A., Pohst, M., and Diaz y Diaz, F.: A table of quintic number fields, Math. Comp. 63 (1994) 361-376. 